\title{
Segregación, estructura y composición social del territorio metropolitano en Santiago de Chile. Complejidades metodológicas en el análisis de la diferenciación social en el espacio ${ }^{1}$
}

\author{
Felipe Link ${ }^{2}$, Felipe Valenzuela ${ }^{3}$ y Luis Fuentes ${ }^{4}$
}

\begin{abstract}
RESUMEN
El estudio de la diferenciación social en el espacio requiere de importantes definiciones teórico-metodológicas que le permitan abordar la compleja relación entre espacio y sociedad. El objetivo de este artículo es presentar una metodología novedosa de análisis de la diferenciación social en el espacio en el Área Metropolitana de Santiago, utilizando tipologías socioespaciales. Los resultados indican que, si bien es posible identificar algunos patrones generales similares entre el uso de tipologías y los resultados de estudios previos basados en el análisis sintético de segregación residencial socioeconómica, la metodología propuesta permite abordar de mejor forma la complejidad social del espacio metropolitano. Además, se pretende relevar la centralidad de la variable ocupacional, entendida como un factor importante de diferenciación social en el contexto urbano, así como en el estudio e interpretación de esta relación asociada a esta metodología.
\end{abstract}

Palabras clave: Estructura socioterritorial, segregación, tipologías, metodología.

\begin{abstract}
The study of social differentiation in space requires significant theoretical and methodological definitions in order to address the complex relationship between space and society. In that regard, it is possible to identify different lines of research that claim to account for this relationship. The aim of this paper is to present an analysis of social differentiation in space in the metropolitan area of Santiago using socio- spatial typologies. The results indicate that, although it is possible to identify some general patterns that are similar when using different approaches, typology analysis can better address the social complexity of metropolitan space. Finally, this paper aims to account for the centrality of the variable occupation as a factor of social differentiation in the urban context.
\end{abstract}

Key words: Socio-territorial structure, segregation, typology, methodology.

\footnotetext{
Este trabajo fue realizado en el contexto del Proyecto CONICYT Anillos SOC1106, el Proyecto Fondecyt de Iniciación $N^{\circ} 11121477$, y el Centro de Estudios de Conflicto y Cohesión Social (COES), CONICYT/FONDAP N 15130009. Artículo recibido el 1 de junio de 2015, aceptado el 10 de julio de 2015 y corregido el 7 de agosto de 2015.
}

2 Instituto de Estudios Urbanos y Territoriales, Pontificia Universidad Católica de Chile (Chile). E-mail: felipe.link@uc.cl

3 Instituto de Estudios Urbanos y Territoriales, Pontificia Universidad Católica de Chile (Chile). E-mail: fevalenzuela@uc.cl

4 Instituto de Estudios Urbanos y Territoriales, Pontificia Universidad Católica de Chile (Chile). E-mail: Ifuentes@uc.cl 
La pregunta por la relación entre espacio y sociedad ha sido permanente en los estudios urbanos, y se ha intentado resolver tanto a nivel teórico como práctico. El presente artículo pretende abordar esta discusión, profundizando en una aproximación metodológica específica basada en la construcción de tipologías socioespaciales, para intentar establecer en qué medida sus resultados son capaces de dar cuenta de mejor forma, de la complejidad de la diferenciación social en el espacio, entendida como la capacidad de dar cuenta de la estructura y composición social de un territorio, más allá de la capacidad de sintetizar indicadores globales o de describir predominios arbitrarios de una categoría determinada en el territorio. Para ejemplificar esta discusión, se analiza el caso del área metropolitana de Santiago, en función de la distribución residencial de las categorías socioocupacionales de su población al año 2002.

Esta tarea se hace cada vez más relevante, sobre todo considerando la complejidad que hoy enfrenta el proceso urbano, en el cual la principal característica es una tendencia renovada de urbanización generalizada, que utiliza al espacio como instrumento de reproducción del capital (Harvey, 1989). Neil Brenner caracteriza recientemente esta tendencia como un

"proceso emergente de urbanización extendida (que) está produciendo una estructura variopinta que, en lugar de concentrarse en puntos nodales o de circunscribirse a regiones delimitadas, se teje ahora de manera desigual y con una densidad cada vez mayor en grandes extensiones de todo el mundo"(Brenner, 2013: 40) $)^{5}$.

Este proceso de urbanización generalizada no es aleatorio en su estructuración. Al contrario, la segregación socioespacial

\footnotetext{
5 Muchos otros autores han dado cuenta del proceso de urbanización generalizada, iniciado por $\mathrm{H}$. Lefebvre en "La Revolución Urbana". En el contexto latinoamericano, Carlos de Mattos (2006) ha insistido en una serie de tendencias constitutivas de la ciudad latinoamericana en la globalización, que siguen el patrón de lo urbano generalizado.
}

aparece como una de las consecuencias e impactos fundamentales en el territorio, lo que remite a la pregunta por la relación entre la estructura del espacio físico y la estructura del espacio social.

Desde una perspectiva marxista, la segregación urbana se entiende como una "distribución de los lugares de residencia (que) sigue las leyes generales de la distribución de los productos $y$, por tanto, produce reagrupaciones en función de la capacidad social de los sujetos, o sea, en el sistema capitalista, en función de sus rentas, de su estatuto profesional, del nivel de instrucción, etc." (Castells, 2004: 203) y eso es un problema de clase. Desde la misma perspectiva, Harvey (1989) señala críticamente que las explicaciones tradicionales a la diferenciación residencial no van más allá del argumento que personas similares prefieren vivir entre sí, lo que no toma en cuenta la relación con elementos estructurales de producción del espacio, y cae en modelos simplistas asociados a la "soberanía del consumidor" o al comportamiento de un "actor racional" capaz de decidir su localización, lo que expresado en el mercado "produciría" diferenciación residencial (Harvey, 1989).

A partir de esta argumentación, se plantea la necesidad de generar nuevos esfuerzos metodológicos para tratar de interpretar las cada vez más complejas relaciones sociales en el espacio, Tal esfuerzo, ha sido realizado recientemente por otros autores. Específicamente, Ruiz-Tagle y López (2014), en relación al uso de diferentes variables para la estratificación social, más allá de la segmentación socioeconómica. Este trabajo, pretende contribuir a esa discusión, específicamente desde una perspectiva metodológica.

Por esto, en los capítulos siguientes se pretende analizar las posibilidades investigativas y metodológicas de esta relación, a través del uso de tipologías socioespaciales y su pertinencia en el contexto de los estudios tradicionales de segregación y ejemplificada a través del caso de Santiago de Chile, entendida como un buen ejemplo de la implementación de políticas neoliberales e impactos socioterritoriales asociados a una nueva fase de modernización capitalista (De Mattos, 2006). 


\section{Alternativas metodológicas para el estudio de la diferenciación social en el espacio}

Los efectos socioterritoriales de la transformación metropolitana contemporánea, tienen que ver básicamente con una distribución residencial desigual de los diferentes grupos sociales, que genera procesos de micro y macrofragmentación urbana (Link, 2010). Como constatan De Mattos et al. (2005) para el caso de Santiago, existen diferentes tendencias en la evolución de la localización territorial de los grupos sociales, analizados según categoría ocupacional. Estas tendencias permiten hablar, por un lado, de una homogeneidad en la localización de los grupos altos, de tendencias hacia la medianización de distritos tradicionalmente populares y de tendencias autosegregativas, que se reflejan en la morfología de esta área metropolitana (De Mattos et al., 2005).

Para comprender estas tendencias, una de las tradiciones de estudio más desarrolladas conceptual y metodológicamente, para analizar la relación entre fenómenos sociales y la configuración del espacio en el contexto urbano corresponde a las investigaciones sobre segregación residencial.

El concepto de segregación residencial ha sido desarrollado ampliamente desde la sociología norteamericana para explicar el deterioro de las condiciones de vida y acceso a las oportunidades, especialmente de la población negra en las ciudades de Estados Unidos (Wilson, 1987; Galster \& Killen, 1995).

La aplicación del concepto de segregación residencial al caso de las ciudades latinoamericanas, y chilenas en particular, ha adoptado ciertos elementos teóricos y metodológicos de la tradición norteamericana, aunque con algunos matices. La principal variación tiene que ver con el atributo que se utiliza para clasificar a la población, pues la mayor parte de los estudios en la región se concentran en el estudio de la segregación residencial según el nivel socioeconómico, ya sea a través del ingreso, el nivel educacional u otras variables (Rodríguez, 2001), dejando de lado diferencias étnicas o raciales.

En este escenario, las diferentes formas de concebir la diferenciación social en la ciudad deriva en distintas aproximaciones metodológicas para llevar adelante su estudio empírico, de manera que, en palabras de Ribeiro (2003) se identifican dos familias metodológicas. Por un lado, las que se traducen en índices sintéticos y por otro, las que utilizan tipologías. En términos generales, la primera de ellas se deriva de la línea de investigación en segregación residencial norteamericana, mientras la segunda tiene una vinculación más directa con las investigaciones sobre estratificación social, buscando dar cuenta de su dimensión territorial en el contexto de las dinámicas de urbanización propias de una economía globalizada.

\section{Análisis de segregación residencial}

Por su parte, la segregación puede ser definida como "el grado de proximidad espacial o de aglomeración territorial de las familias pertenecientes a un mismo grupo social, sea que este se defina en términos étnicos, etarios, de preferencias religiosas o socioeconómicos" (Sabatini et al., 2001: 27), la manifestación "objetiva" de la segregación residencial se produce de acuerdo a dos dimensiones: 1) la tendencia de los grupos sociales a concentrarse en algunas áreas de la ciudad y 2) la conformación de áreas o barrios socialmente homogéneos (Sabatini y Sierralta, 2006). Es decir, existe segregación residencial como resultado de la presencia de grupos sociales que se localizan preferentemente en algunas zonas de la ciudad por sobre otras, y también por la existencia de zonas dentro de la ciudad donde la proporción de miembros de uno u otro grupo social es superior al promedio, conformándose de esa manera espacios de homogeneidad social. Además de estas manifestaciones objetivas de la segregación residencial, Sabatini et al. (2001) consideran dentro de la definición del fenómeno una tercera dimensión, que tiene que ver con la percepción subjetiva que tienen de la segregación "objetiva" las personas que la experimentan. En este contexto, desde los índices sintéticos, la medición se ha realizado priorizando la primera de sus dimensiones, asumiendo la supuesta 
existencia de un grado importante de correlación entre ambas (Sabatini y Sierralta, 2006).

Para medir la primera dimensión objetiva de la segregación, el indicador más utilizado ha sido el índice de disimilitud de Duncan, desarrollado y utilizado principalmente para medir segregación racial (Rodríguez, 2001). El índice Duncan constituye un indicador sintético de la relación que existe entre la composición social de las subunidades territoriales (barrios, distritos censales, etc.) y la composición social de la unidad territorial de orden superior (la ciudad o área metropolitana). Este indicador adquiere valores de 0 (ausencia de segregación) a 1 (máxima segregación) y se interpreta como el porcentaje de miembros del grupo minoritario que debería cambiar su lugar de residencia para alcanzar una situación de ausencia de segregación (Rodríguez, 2001: 22). Una de las desventajas del índice Duncan es que trabaja con variables dicotómicas, por lo que se adapta mejor a la medición de la segregación racial (donde el objetivo puede estar puesto en la distinción blanco-negro) que a la medición de la segregación socioeconómica, de mayor relevancia en América Latina (Rodríguez, 2001). Para enfrentar este problema, se debe definir algún criterio de corte sustantivo que permita distinguir dos grupos socioeconómicos. De esta forma, como variable estratificadora para la medición de la segregación, algunos autores han considerado los años de estudio utilizando como criterio de corte los 12 años de escolaridad (Arriagada y Morales, 2006), mientras otros realizan la medición a través de los Grupos Socio-Económicos (GSE) provenientes de los estudios de mercado (Sabatini et al., 2008).

Otro indicador que ha sido utilizado para dar cuenta de la segregación residencial es el índice de Moran. Aunque en la literatura se ha destacado como un índice espacial, a diferencia del caso anterior que no considera el espacio como una variable (Garrocho \& Campos-Alanís, 2013); el índice de Moran no ha sido encasillado en ningunas de las dimensiones de la segregación. Estos índices espaciales de segregación, surgen aceleradamente con el desarrollo de la estadística espacial desde los años noventa (Anselin, 1995). El índice global de Moran (Bailey \&
Gatrell, 1995), mide la proximidad entre unidades territoriales donde residen categorías de población con características similares, esto es, indica si la distribución de los datos en el espacio se autocorrelacionan entre sí presentando un patrón no aleatorio. Definida de manera simple, la autocorrelación espacial es la concentración o dispersión de los valores de una variable en un determinado lugar. El diseño de este índice es similar al coeficiente de correlación de Pearson, ya que sus valores varían entre +1 y -1 , en donde el primer valor significa una autocorrelación positiva perfecta (perfecta concentración), y el segundo significa una autocorrelación negativa perfecta (perfecta dispersión); el cero significa un patrón espacial totalmente aleatorio.

La confiabilidad de este indicador es sensible al número de entidades espaciales que se analizan, que debe superar las treinta observaciones. Se trata de un indicador que no describe la composición social interna de las diferentes zonas que se analizan, sino más bien la predominancia y su correlación con las zonas vecinas. Además, se debe considerar que constituye un indicador para toda una ciudad, lo cual de cierta manera lo asemeja a los indicadores no espaciales de segregación residencial. Las ventajas han sido ampliamente estudiadas y contrastadas con los índices de segregación no espaciales (Garrocho \& Campos-Alanís, 2013). Específicamente, gracias al desarrollo de los sistemas de información geográfica (SIG) el potencial de estas técnicas está relacionado a la posibilidad de analizar los aspectos eminentemente "espaciales" de los datos, como el caso de Local Moran (Anselin, 1995) o LISA (Local Indicator of Spatial Association). El coeficiente Lisa es equivalente al cálculo del Moran para cada una de las subunidades espaciales, permitiendo evaluar la significación estadística de las correlaciones entre las subunidades vecinas en los patrones locales de agrupamiento. Ambos índices incorporan la noción de "vecindad" mediante las pruebas de autocorrelación (Anselin, 1995). De acuerdo a sus características metodológicas, a nuestro juicio estos índices espaciales (Moran y Lisa), se relacionan en mayor medida con la primera dimensión de la segregación, es decir la concentración espacial. 
Tanto Moran como LISA han sido utilizadas para estudiar la segregación en distintas ciudades como Santiago, Lima y Bogotá (Fuentes, 2012), Barcelona (Martori, 2007), Montevideo (Kaztman \& Retamoso, 2006), Buenos Aires (Groisman \& Suárez, 2006), entre otras. Para el caso específico de la ciudad de Santiago de Chile, Fuentes (2012) plantea una estructura de localización correlacionada entre los diferentes grupos sociales, analizados según categoría ocupacional. Todos los valores del índice global Moran calculados en este caso tienen un puntaje mayor a 0,5. Los valores más altos, es decir, de mayor correlación, corresponden a los grupos directivos y los grupos obreros, con valores superiores a 0,7. Mientras, los grupos medios obtienen los menores valores de correlación espacial.

En cuanto a la segunda dimensión de la segregación residencial, tradicionalmente vinculada a las consecuencias negativas del fenómeno, su medición se ha realizado a través de diferentes indicadores. Una alternativa es el índice de aislamiento, que "mide la probabilidad de una familia de un determinado grupo de encontrarse en el área que habita con gente de su misma condición social" (Sabatini et al., 2008). Una versión inversa del mismo es el índice de exposición, que mide la probabilidad de que sujetos de dos grupos sociales diferentes se encuentren en el espacio residencial (Echazarra, 2010). Sin embargo, una de las desventajas de estos índices es que tienden a ser muy sensibles al tamaño de cada uno de los grupos sociales, de manera que, por ejemplo, los grupos más numerosos serán generalmente los de mayor aislamiento, pues tendrán mayor probabilidad de encontrarse con otros miembros del mismo grupo en su espacio residencial. Por otro lado, ambos índices funcionan como un indicador sintético, es decir, como un valor único por grupo para toda la ciudad, lo que resulta una desventaja cuando se pretende realizar una caracterización a menor escala. Al tratarse de un atributo de las subunidades territoriales (por ejemplo, los barrios), generalmente la medición de la segunda dimensión de la segregación se realiza intentando describir de manera independiente a cada una de las subunidades, por ejemplo mediante un indicador de homogeneidad/ heterogeneidad social del espacio, recurriendo en algunos casos a medidas estadísticas de dispersión como la desviación estándar (Sabatini et al., 2001) o el coeficiente de variación (Rodríguez y Arriagada, 2003). No obstante, ambas medidas requieren de una variable de intervalo (como los ingresos o los años de escolaridad) para ser utilizadas, lo cual no siempre corresponde a la naturaleza de las variables con las que se puede medir la segregación, donde se presume existen diferencias sustantivas entre un grupo y otro por ejemplo, los grupos socioeconómicos o grupos socioocupacionales. De esta forma, para dar cuenta del nivel de homogeneidad social de los barrios algunos autores utilizan simplemente el porcentaje de hogares del barrio que presentan la característica de interés, como por ejemplo, el porcentaje de hogares pertenecientes a alguno de los grupos socioeconómicos (Sierralta, 2008), asumiendo que mientras mayor sea la presencia del grupo, mayor es la homogeneidad social de ese espacio. Este tipo de resultado puede apoyarse en la presentación de mapas, los que permiten expresar territorialmente y de manera gráfica las diferencias en la distribución de los grupos sociales en la ciudad.

La mayor parte de las investigaciones realizadas en Chile respecto al tema de la diferenciación social del espacio corresponden a estudios que se inscriben en la tradición de la segregación residencial en su versión socioeconómica y tienen como principales resultados tendencias generales para el caso de Santiago, donde se ha constatado que sus niveles de segregación -medida de acuerdo a indicadores de disimilitud, que refieren a la descrita primera dimensión de la segregación- son relativamente altos en el contexto latinoamericano, aunque muy por debajo de lo observado en Estados Unidos (Sabatini y Sierralta, 2006; Rodríguez y Arriagada, 2003). La tendencia de las últimas décadas parece ser hacia una estabilización o leve reducción de la segregación residencial en Santiago, entendida geográficamente (Rodríguez, 2007 y 2001; Sabatini y Sierralta, 2006). Además, de acuerdo a estas investigaciones, la segregación residencial estaría modificando su patrón en las ciudades latinoamericanas, tendiendo a disminuir debido principalmente a un proceso de dispersión espacial de la elite, posibilitado por un mayor dinamismo inmobiliario (Sabatini et al., 2008). Por otro lado, los estudios 
de Rodríguez (2007; 2001) indican que si se estudia la segregación residencial en períodos más largos (por ejemplo el AMS entre 1982 y 2002) efectivamente se observa una reducción de la segregación, pero se comprueba que esta disminución no se debe a una migración intrametropolitana desde comunas ricas a comunas más pobres (hipótesis de la reducción de la segregación), sino a la expansión educativa que hace aumentar los años de escolaridad sobre todo en los grupos más bajos. En este sentido, siguiendo a Ortiz y Escolano (2013:94) vemos que

"Ios movimientos residenciales y los destinos selectivos de los migrantes en función de su formación y ocupación, han contribuido notablemente a la modificación a todas las escalas de los modelos de segregación socioespacial preexistentes en el Gran Santiago (...) consolidando el espacio social del cono oriente, producto de la alta calificación ocupacional y educacional de los migrantes del propio sector".

Por otra parte, Hidalgo (2004) observa que en las últimas décadas se produce una modificación en la estructura urbana del AMS debido a la instalación de condominios cerrados para población de ingresos medios y altos que, en la medida en que crecen en su escala, comienzan a instalarse en comunas periféricas tradicionalmente habitadas por población de menores recursos. Esta situación efectivamente acerca geográficamente a ricos y pobres, pero considerando la tendencia hacia el "enrejamiento" que caracteriza a estos conjuntos residenciales, ello no necesariamente genera integración social.

En definitiva, existe evidencia acumulada respecto a la evolución de la segregación residencial en el AMS entendida desde diferentes perspectivas, que permite sostener que esta se ha transformado durante las últimas décadas, aunque no existe consenso respecto a los efectos que ello tiene en términos de integración social, ni de las causas de estas tendencias, pudiendo atribuirse tanto a ciertos procesos migratorios intrametropolitanos ("dispersión de la elite") como a factores relacionados a las variaciones que experimenta en el tiempo la distribución de las variables con que se mide la segregación residencial.
Además, es importante tener en cuenta que este enfoque ha sido sometido a crítica recientemente debido a diferentes limitaciones asociadas a los modelos de estratificación comúnmente utilizados, las características de los indicadores seleccionados, y las interpretaciones de los resultados en términos de los problemas sociales derivados de la segregación y las respuestas de política pública que se extraen de los mismos (Ruiz-Tagle y López, 2014).

\section{Construcción de tipologías socio- espaciales}

La construcción de tipologías constituye una segunda aproximación metodológica al estudio de la diferenciación social en el espacio urbano. Siguiendo a Ribeiro (2003), la tipología socioespacial es un instrumento de clasificación y descripción que pretende caracterizar socialmente las áreas geográficas de la ciudad. La aplicación de este método permite agrupar las unidades espaciales de acuerdo a un conjunto de variables consideradas centrales en la descripción o interpretación de los principios de división del espacio, donde cada tipo reúne las unidades geográficas con valores idénticos o muy similares. A pesar de los avances expuestos anteriormente en relación a la tradición de medición de la segregación residencial, la gran diferencia con el enfoque asociado a la construcción de tipologías socioespaciales tiene que ver con la posibilidad de caracterizar de forma conjunta la diversidad interna del territorio y explorar la estructura latente, a partir de su diferenciación social. Además, las tipologías en relación con otras variables complementarias, permiten identificar los espacios donde ocurre una acumulación de desigualdades que pueden identificar situaciones de exclusión e integración socioespacial. Permiten también, la identificación de los procesos de transformación de la estructura urbana a partir de su representación en mapas temáticos (Ribeiro, 2003).

La construcción de tipologías que aquí se propone requiere de la realización previa de un análisis factorial de correspondencias (AFC), un procedimiento estadístico que tiene como objetivo explicar la estructura de las covariaciones entre variables, mediante la definición de una cierta cantidad de factores. El 
método se aplica para descubrir dimensiones latentes y una estructura subyacente a los datos, que permitan interpretar manifestaciones visibles (Buzai, 2003). Una vez realizado el AFC, es posible avanzar en la caracterización, a través de un método de clasificación jerárquica y de agrupamiento o construcción de clases de unidades homogéneas internamente y heterogéneas entre sí, lo que finalmente genera las tipologías socioespaciales posibles de ser descritas estadísticamente y representadas cartográficamente mediante SIG.

Este tipo de metodología es similar al implementado desde la tradición de estudios de "ecología factorial", desarrollados con el objetivo de identificar áreas sociales a partir de un análisis factorial. Esta línea de estudios se basa principalmente en el trabajo de Shevky y Bell (1955), quienes sostienen que la estructura social de una ciudad puede ser descrita en términos de tres factores: rango social o estatus económico, urbanización o estatus familiar, y segregación o estatus étnico. De esta forma, es posible identificar diferentes áreas sociales en la ciudad, las cuales se conforman por unidades espaciales que son similares entre sí en función de los tres factores establecidos. Inspiradas en el trabajo de Shevky y Bell, distintas investigaciones buscaron identificar los factores de diferenciación del espacio social, aplicando análisis factorial para dilucidar estas dimensiones latentes (Ortiz y Schiappacasse, 2000). Sin embargo, a diferencia de estos estudios, que por lo general consideraron una gran cantidad de variables en el procesamiento estadístico, en la propuesta de tipologías socioespaciales que aquí se propone se utiliza solamente la variable ocupacional y se busca profundizar principalmente en la caracterización de los grupos que se generan a través de un análisis de clasificación.

\section{Estructura y composición socioterritorial en el Área Metropolitana de Santiago}

A partir de lo señalado hasta aquí, en relación a las limitaciones de las medidas tradicionales de la segregación, en este capítulo presentamos un análisis del AMS, a partir de tipologías, con el objetivo de ampliar las posibilidades metodológicas de la diferenciación social en el espacio y dar cuenta de la estructura y composición socioterritorial, incluyendo la variable ocupacional como una variable operacional de esta diferenciación.

La categoría ocupacional aparece como una variable clave para el análisis de la estructura urbana. Siguiendo a Preteceille y Ribeiro (1999) y a De Mattos et al. (2005) entendemos que en el contexto de globalización y reestructuración económico-productiva, las transformaciones en la estructura social, son una de las causas principales de las transformaciones espaciales y urbanas. Más aún, en palabras de Ribeiro (2003:97):

\footnotetext{
"la selección de la clasificación socioocupacional supone que la estratificación social por la ocupación, localiza a los individuos en posiciones sociales asociadas a un conjunto de propiedades más o menos convergentes. Esto torna a la ocupación como un indicador importante de identidad social y de posesión de recursos en términos de cantidad y calidad (capital económico, cultural, social y simbólico). Por lo tanto, la utilización de este indicador permite discriminar la localización de los individuos en el espacio como resultado de la posesión de recursos, de orientaciones o preferencias y de restricciones. Este criterio tiene un papel unificador de las múltiples dimensiones implicadas en la distribución de las personas en el espacio urbano".
}

La variable ocupacional ha sido ampliamente utilizada en estudios de estratificación social, teniendo en cuenta que las diferencias en el trabajo constituyen el punto central de la diferenciación social, y tienden a implicar también diferencias en términos de nivel educativo, estatus, ingresos y otros. En este sentido, una de las principales ventajas de la utilización de esta variable es que en muchos casos al conocer la información respecto a la ocupación, se pueden asumir varios datos adjuntos sobre otras dimensiones de la diferenciación social (Mac-Clure et al., 2014). Junto con ello, las posibilidades de obtener información detallada respecto a la ocupación a través de una encuesta u otro instrumento similar son mucho mayores que cuando se intenta recabar otro tipo de datos como los 
ingresos, donde el nivel de rechazo tiende a ser importante. Sin embargo, la variable ocupacional implica también algunos problemas, como las dificultades para clasificar a estudiantes, jubilados e incluso trabajadores que se encuentran fuera del mercado laboral formal (Barozet, 2007).

En suma, teniendo en cuenta las ventajas y desventajas asociadas a su utilización, la variable ocupacional resulta pertinente para un estudio sobre la estructura y composición socioterritorial en el contexto metropolitano. Para trabajar con esta variable, existen variadas herramientas de clasificación de las ocupaciones, entre las que se encuentra la Clasificación Internacional Uniforme de Ocupaciones, en su versión del año 1988 (CIUO-88), que fue la utilizada en Chile para el Censo de Población y Vivienda 2002, que constituye la fuente de datos para el presente estudio. Esta clasificación consiste en una herramienta para organizar los diferentes tipos de trabajo en función de las tareas que implica cada uno de ellos. A través de la descripción de cada trabajo, se categorizan las ocupaciones en diferentes grupos, cuyo ordenamiento puede ser entendido de manera jerárquica entre las ocupaciones que requieren mayores competencias e implican un mayor grado de autoridad y/o autonomía en el proceso de trabajo, y aquellas ocupaciones que no requieren calificación. En el Cuadro $\mathrm{N}^{\circ} 1$ se observa el listado de los grandes grupos de esta clasificación, los cuales fueron utilizados para construir las tipologías socioespaciales del AMS.

\section{Estructura social del territorio metropolitano en el AMS}

Los resultados del análisis de tipologías socioterritoriales basadas en las ocupaciones, indican que la estructura socioespacial

Cuadro $\mathrm{N}^{\circ} 1$

Categorías ocupacionales según grandes grupos CIUO

\begin{tabular}{|c|l|l|}
\hline $\begin{array}{c}\text { Gran } \\
\text { Grupo* }\end{array}$ & \multicolumn{1}{|c|}{ Nombre } & \multicolumn{1}{|c|}{ Nombre abreviado } \\
\hline 1 & $\begin{array}{l}\text { Miembros del poder ejecutivo y de los cuerpos } \\
\text { legislativos y personal directivo de la administra- } \\
\text { ción pública y de empresas }\end{array}$ & Dirigentes (DIR) \\
2 & $\begin{array}{l}\text { Profesionales, científicos e intelectuales } \\
\text { Técnicos y profesionales de nivel medio }\end{array}$ & Profesionales (PRO) \\
4 & $\begin{array}{l}\text { Empleados de oficina } \\
\text { Técnicos (TEC) }\end{array}$ \\
5 & $\begin{array}{l}\text { Trabajadores de los servicios y vendedores de } \\
\text { comercios y mercados }\end{array}$ & Oficinistas (OFI) \\
6 & $\begin{array}{l}\text { Agricultores y trabajadores calificados agrope- } \\
\text { cuarios y pesqueros }\end{array}$ & Servicios (SER) \\
7 & $\begin{array}{l}\text { Oficiales, operarios y artesanos de artes mecáni- } \\
\text { cas y de otros oficios }\end{array}$ & Oprícolas (AGR) \\
8 & $\begin{array}{l}\text { Operadores de instalaciones y máquinas y mon- } \\
\text { tadores }\end{array}$ & Operadores y conductores (OPC) \\
9 & $\begin{array}{l}\text { Trabajadores no calificados } \\
0\end{array}$ & Fuerzas Armadas \\
\hline
\end{tabular}

Fuente: Elaboración propia en base a OIT. http://www.ilo.org/public/spanish/bureau/stat/isco/isco88/major.htm * Se excluye del análisis, la categoría Fuerzas Armadas, ya que se aleja demasiado de un patrón de distribución y dificulta la conformación de grupos. 
Cuadro $\mathrm{N}^{\circ} 2$

Contribución de las categorías ocupacionales a la conformación de ejes de AFC (AMS 2002)

\begin{tabular}{|l|r|r|}
\hline Categoría ocupacional & \multicolumn{1}{|c|}{ Eje 1 } & \multicolumn{1}{|c|}{ Eje2 } \\
\hline Dirigentes & 5,61 & 9,86 \\
Profesionales & 54,36 & 4,21 \\
Técnicos & 5,96 & 18,12 \\
Oficinistas & 0,06 & 19,75 \\
Servicios & 2,93 & 4,7 \\
Agrícolas & 0,89 & 5,23 \\
Operarios calificados & 16,47 & 0,45 \\
Operadores y conductores & 6,27 & 2,6 \\
Trabajadores no calificados & 7,44 & 35,08 \\
\hline
\end{tabular}

Fuente: Elaboración propia.

del área metropolitana de Santiago, a nivel de zonas censales, basado en un Análisis Factorial de Correspondencias simples (AFC) es principalmente una estructura jerárquica y en segundo lugar, una estructura matizada por la mixtura social de clases medias en el territorio. La construcción de tipologías socioespaciales da cuenta, como se mencionó más arriba, de la forma y contenido de la distribución social en el espacio, caracterizando la complejidad de la relación entre el espacio físico y el espacio social en la ciudad.

Este tipo de análisis se centra en la interpretación de los patrones de distribución de las unidades territoriales en un momento determinado ${ }^{6}$, así como en los resultados de la clasificación y construcción estadística de tipologías socioespaciales a partir de las ocupaciones.

\footnotetext{
6 En este caso el año 2002 para un área metropolitana de 34 comunas (provincia de Santiago, más las comunas de San Bernardo y Puente Alto). Esto es importante, ya que un análisis actualizado de esta estructura debería considerar un área metropolitana mayor, como es el caso del Sistema Urbano Metropolitano de Santiago (SUMS) descrito en De Mattos et al. (2014) y que agrupa 47 comunas de la Región Metropolitana. Sin embargo, no hay disponibilidad de datos actualizados para esta escala y con datos al año 2002 no es coherente ampliar la base territorial, ya que no se cumplen los criterios que Ilevan a una modificación de escala.
}

En este análisis, las unidades o individuos, corresponden a zonas censales. Cada zona se distribuye en el plano según el peso de cada categoría socioocupacional, lo que permite describir la forma de dicha distribución basada en el trabajo.

Los dos primeros ejes o factores, en la estructura del AMS, explican el 88,59\% de la variación total de la distribución centrada en las ocupaciones. Es decir, que el análisis de dichos factores es suficiente y muy importante para entender la estructura urbana en función de esta variable.

Por otra parte, como se puede observar en el Cuadro $\mathrm{N}^{\circ} 2$, las mayores contribuciones a la conformación del primer eje corresponden a las categorías: profesionales, operarios calificados, trabajadores no calificados. Mientras, al segundo eje contribuyen principalmente: dirigentes, técnicos, oficinistas y empleados, trabajadores no calificados.

Si tomamos en cuenta las coordenadas de cada una de estas categorías, vemos que se oponen en el plano factorial como se observa en la Figura $\mathrm{N}^{\circ} 1$. Como se puede observar, el primer eje horizontal o factor número 1, separa las categorías profesionales, dirigentes y en menor medida técnicos, de todo el resto. Mientras el segundo factor o eje vertical, separa a dirigentes, trabajadores no calificados, trabajadores agrícolas y en menor medida profesionales, de todo el resto. Al contrastar 
Figura $\mathrm{N}^{\circ} 1$

AFC categoría ocupacional AMS 2002

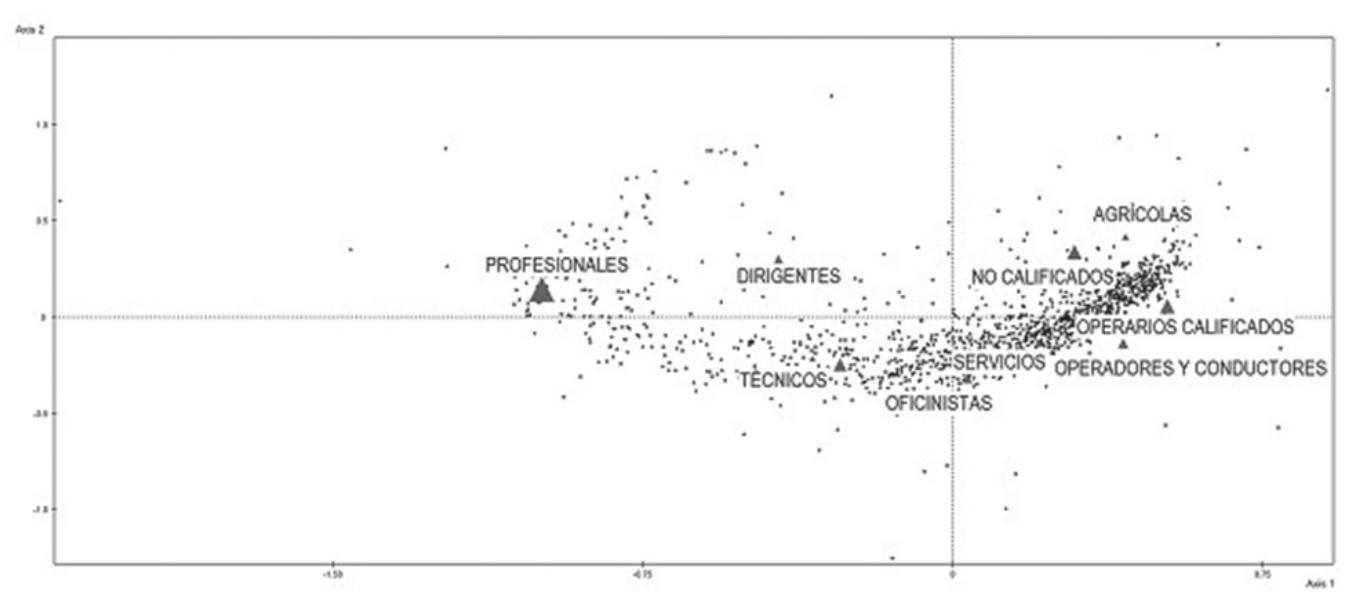

Fuente: Elaboración propia en base a datos censales de 2002.

el gráfico de la Figura $\mathrm{N}^{\circ} 1$ con la tabla de contribuciones a cada factor (Cuadro $N^{\circ} 2$ ), vemos que el primer eje, separa zonas de mayor jerarquía ocupacional, como son los dirigentes, profesionales y técnicos, de zonas de menor jerarquía ocupacional, configurando la estructura social del territorio metropolitano de Santiago.

El segundo factor agrupa a los dos polos de la jerarquía, separándolos del resto de las categorías. Es decir, que el patrón que sigue la distribución espacial a nivel de zonas censales en función de las categorías ocupacionales tiende a agrupar por un lado, a las categorías superiores e inferiores. Por otro, tiende a agrupar los polos y separarlos de los sectores medios. De acuerdo a este resultado, se puede sostener que algunas áreas en la estructura urbana se presentan de forma polarizada, es decir, concentran a los polos de la jerarquía ocupacional, mientras que la gran mayoría de las áreas en la estructura urbana concentra grupos medios con diferente composición interna. Bajo esta interpretación, tanto polarización y medianización de la estructura urbana y social de Santiago son posibles y de hecho ocurren paralelamente.

\section{Composición social del territorio metropolitano en el AMS}

Para avanzar en la hipótesis anterior, a continuación se presentan los resultados de la construcción de tipos o grupos generados por el análisis de clasificación. La clasificación por tipologías estadísticas, a partir de los análisis factoriales, supone distintos niveles de agrupación. Estos niveles son los que muestra el gráfico denominado dendograma, presentado en la Figura $N^{\circ} 2$. Este esquema considera un rango de variabilidad en la distribución y heterogeneidad de las unidades. Así, es posible agrupar todas las zonas censales en un solo grupo, entendidas por ejemplo como característica común, el hecho que todas pertenecen al AMS. Luego, el polo opuesto o rango en la clasificación, es considerar a cada zona como una unidad diferente. En esta lógica, tenemos la posibilidad de encontrar un grupo o $\mathrm{n}$ grupos (número total de unidades). En ese rango, el análisis de clasificación, representado por el dendograma, ofrece múltiples posibilidades intermedias, de acuerdo al grado de homogeneidad interna y heterogeneidad externa de las unidades en función de las variables sometidas al análisis. Por lo tanto, el dendograma ofrecerá la posibilidad de considerar dos, tres, cuatro o más grupos intermedios entre $1 \mathrm{y} \mathrm{n}$.

Para el caso del AMS en 2002, este dendograma muestra las diferentes posibilidades de corte en la generación de grupos. El corte óptimo considerado en este caso es en seis grupos. Los criterios para seleccionar el nivel de corte, tienen que ver por un lado con coherencia teórica e interpretativa y por otro, 


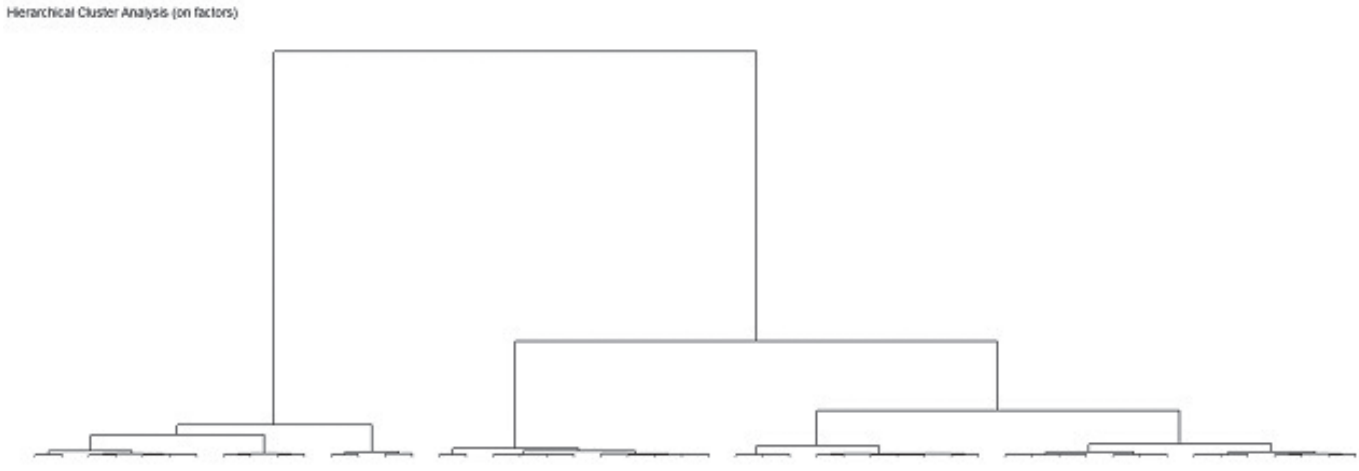

Fuente: Elaboración propia en base a datos censales de 2002.

con criterios estadísticos de varianza inter e intragrupos. La idea de clasificar, es la de reunir elementos los más homogéneos posible entre sí y heterogéneos del resto. Esto se dimensiona con el coeficiente de variación inter grupo, que en este caso es superior al $75 \%$.

Los tipos empíricos generados por el análisis de clasificación se ubican en el plano factorial de la estructura socioterritorial mostrada en la Figura $N^{\circ} 3$, y tienen las siguientes características:

El grupo 1 corresponde a la tipología de mayor jerarquía en términos de su concentra- ción y homogeneidad territorial. Si bien agrupa solo a un $2,2 \%$ de las zonas censales del AMS concentra al 8,5\% de todos los dirigentes del AMS y al 7,5\% de todos los profesionales. En su composición interna es un grupo bastante homogéneo, ya que está compuesto prácticamente en un 50\% por dirigentes y profesionales. Es interesante que este grupo está configurado además por trabajadores no calificados, quienes representan un $30 \%$ del grupo, a pesar de que evidentemente no están concentrados ahí, ya que solo un 4,8\% de todos los trabajadores no calificados del AMS reside en estas áreas. Las zonas características de este grupo se localizan principalmente en las comunas de Vitacura y Lo Barnechea.

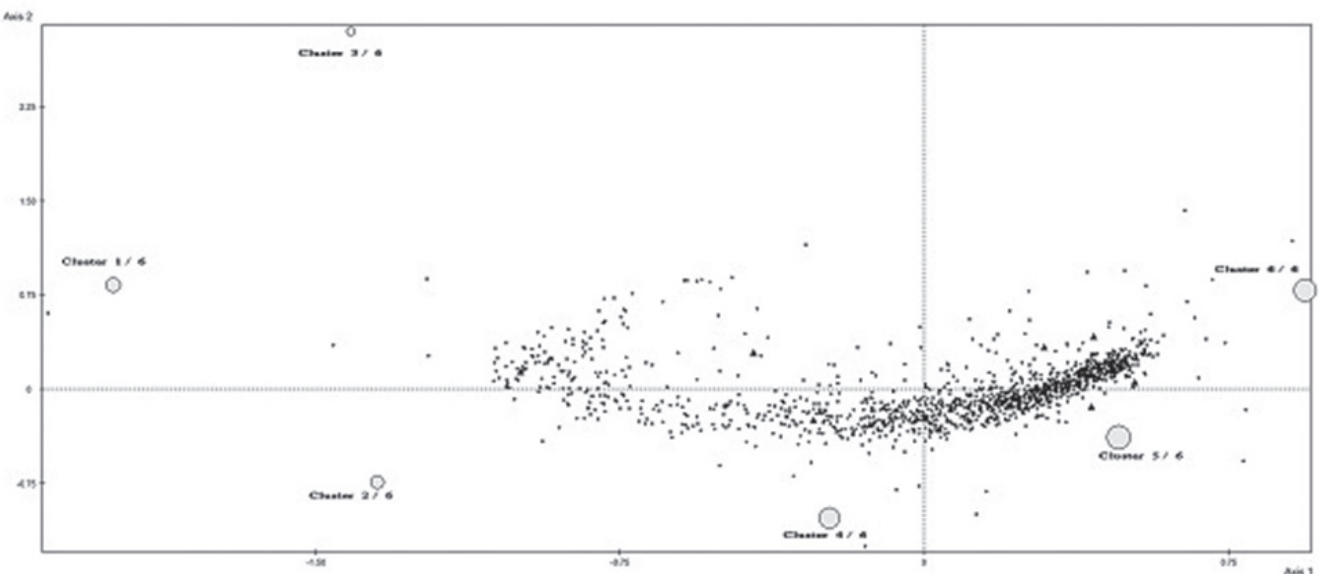

Fuente: Elaboración propia en base a datos censales de 2002. 
El grupo 2 está caracterizado por profesionales, dirigentes y técnicos. Se trata también de un grupo bastante homogéneo y concentrado en cuanto a las categorías más altas de la jerarquía ocupacional, aunque incorpora a los grupos técnicos como constitutivos de la tipología. Agrupa al 8\% del total de zonas del AMS y está compuesto por un $37,1 \%$ de profesionales, $13,3 \%$ de dirigentes, $22 \%$ de técnicos, lo que supera ampliamente la distribución promedio del AMS, como se puede observar en los Cuadros $\mathrm{N}^{\circ} 3$ y $\mathrm{N}^{\circ} 4$. Las zonas representativas del grupo 2 en el AMS se encuentran principalmente en las comunas de Las Condes y Providencia, con presencia también en Peñalolén, La Florida y Huechuraba. Es decir, se trata de un grupo relativamente homogéneo en su composición y donde las categorías altas de la jerarquía ocupacional están concentradas. Por ejemplo, cerca del $35 \%$ de todos los profesionales del AMS se encuentran en este grupo, así como más del $20 \%$ de todos los dirigentes. Esto es importante, ya que esta proporción se distribuye en solo el $8 \%$ del territorio metropolitano, medido a través de zonas censales, lo que concentra las posibilidades de encuentro con habitantes de la mayor jerarquía socioocupacional.

El grupo 3 está caracterizado por profesionales, técnicos, oficinistas y en menor medida dirigentes. La composición interna del grupo es heterogénea en el nivel alto de la jerarquía ocupacional, distribuyendo aproximadamente $26 \%$ para profesionales, $26 \%$ técnicos, $12,5 \%$ oficinistas y cera de $8 \%$ de dirigentes.

Se trata básicamente de un grupo asociado a una clase media calificada, aunque heterogénea, con presencia importante en el centro de Santiago y en los ejes asociados al proceso de medianización de la ciudad (De Mattos et al., 2005), como se observa en la Figura $\mathrm{N}^{\circ} 4$.

El grupo 4 está compuesto y caracterizado principalmente por técnicos, oficinistas y trabajadores del sector servicios, además de algunos profesionales y operadores y conductores. Es decir, se trata de una clase media tradicional en términos de su ocupación, siguiendo los modelos de estratificación social aplicados para el caso de Chile (León y Martínez, 2007; Torche y Wormald, 2007; Barozet, 2007). A pesar de su heterogeneidad interna, en el sentido que solo un grupo (técnicos) supera el $20 \%$ en la distribución del clúster, este parece ser un grupo bastante concentrado. Como se puede observar en el Cuadro $N^{\circ} 4$ cerca del $30 \%$ de todos los técnicos y oficinistas del AMS se encuentran en este grupo. Ahora, esto es así, ya que grupo 4 corresponde al $23 \%$ del territorio metropolitano, por lo que la extensión geográfica es mucho mayor que los grupos precedentes. El grupo 4 se distribuye en zonas principalmente pertenecientes a comunas como Maipú y Puente Alto, además de las comunas del pericentro del AMS y en zonas que siguen las vías estructurantes hacia el sur del área metropolitana (San Miguel, La Cisterna, Estación Central, Macul, etc.). La composición de este clúster es muy similar a la composición media del AMS.

El grupo 5 corresponde principalmente a un grupo socioterritorial obrero tradicional. Está constituido en porcentajes en torno al $15 \%$ de operadores y conductores, operarios calificados y trabajadores de servicios, además de cerca de un $12 \%$ de oficinistas. Concentra cerca de un 35\% de la distribución de cada categoría, principalmente de categorías medias y obreras, aunque es el de mayor extensión territorial, considerando el 32\% del territorio metropolitano. La zona característica corresponde a localizaciones tradicionales fuera del cono de alta renta del AMS.

Por último, el grupo 6 generado en el análisis de clasificación, representa a la base de la jerarquía ocupacional. Está caracterizado por las categorías de trabajadores no calificados y operarios. Además, prácticamente no hay profesionales en el grupo. Corresponde al $26,9 \%$ de las zonas del AMS, concentrando su distribución en zonas pertenecientes a las comunas de Quilicura, Renca, El Bosque, San Ramón, Lo Espejo y La Pintana, entre otras, es decir, las zonas tradicionalmente más vulnerables del AMS, por ejemplo, cerca del $45 \%$ de todos los operarios y trabajadores no calificados del AMS están aquí, concentrando gran parte de los efectos colaterales del desarrollo metropolitano.

La representación en el territorio mediante SIG, de los seis tipos socioterritoriales 
Cuadro $\mathrm{N}^{\circ} 3$

Homogeneidad social del territorio metropolitano de Santiago: porcentaje que representa cada categoría ocupacional dentro de cada clúster

\begin{tabular}{|c|c|c|c|c|c|c|c|c|c|c|c|}
\hline Homogeneidad & $\begin{array}{l}\text { DIR } \\
(\%)\end{array}$ & $\begin{array}{c}\mathrm{PRO} \\
(\%)\end{array}$ & $\begin{array}{l}\text { TEC } \\
(\%)\end{array}$ & $\begin{array}{l}\text { OFI } \\
(\%)\end{array}$ & $\begin{array}{l}\text { SER } \\
(\%)\end{array}$ & $\begin{array}{c}\text { OCF } \\
(\%)\end{array}$ & $\begin{array}{c}\text { OPC } \\
(\%)\end{array}$ & $\begin{array}{c}\text { TNC } \\
(\%)\end{array}$ & $\begin{array}{c}\text { AGR } \\
(\%)\end{array}$ & Total & $\begin{array}{c}\text { Porcentaje de } \\
\text { Zonas censales } \\
\text { del AMS }\end{array}$ \\
\hline Clúster 1 & 19,6 & 29,5 & 11,9 & 2,8 & 3,8 & 1,1 & 0,6 & 29,7 & 1,0 & 100 & 2,2 \\
\hline Clúster 2 & 13,4 & 37,2 & 22,0 & 6,5 & 6,9 & 1,4 & 0,9 & 11,5 & 0,3 & 100 & 8,0 \\
\hline Clúster 3 & 7,9 & 26,6 & 26,8 & 12,6 & 11,7 & 4,2 & 3,4 & 6,4 & 0,3 & 100 & 7,9 \\
\hline Clúster 4 & 5,9 & 11,9 & 22,8 & 14,8 & 15,5 & 9,7 & 7,9 & 10,9 & 0,6 & 100 & 23,0 \\
\hline Clúster 5 & 5,0 & 5,4 & 15,8 & 12,5 & 16,7 & 15,5 & 10,3 & 17,9 & 0,8 & 100 & 32,0 \\
\hline Clúster 6 & 4,9 & 2,5 & 9,5 & 8,2 & 15,6 & 20,4 & 9,7 & 27,4 & 1,7 & 100 & 26,9 \\
\hline $\begin{array}{l}\text { Distribución de } \\
\text { los grupos en el } \\
\text { AMS }\end{array}$ & 6,7 & 11,7 & 16,9 & 10,9 & 14,3 & 12,9 & 7,8 & 17,9 & 0,9 & 100 & 100 \\
\hline
\end{tabular}

Fuente: Elaboración propia en base a datos censales de 2002.

\section{Cuadro $\mathrm{N}^{\circ} 4$}

Concentración social en el territorio metropolitano de Santiago: distribución de las categorías ocupacionales entre los diferentes clúster

\begin{tabular}{|l|r|r|r|r|r|r|r|r|r|r|r|}
\hline Concentración & $\begin{array}{c}\text { DIR } \\
(\%)\end{array}$ & $\begin{array}{c}\text { PRO } \\
(\%)\end{array}$ & $\begin{array}{r}\text { TEC } \\
(\%)\end{array}$ & $\begin{array}{r}\text { OFI } \\
(\%)\end{array}$ & $\begin{array}{r}\text { SER } \\
(\%)\end{array}$ & $\begin{array}{r}\text { OCF } \\
(\%)\end{array}$ & $\begin{array}{c}\text { OPC } \\
(\%)\end{array}$ & $\begin{array}{c}\text { TNC } \\
(\%)\end{array}$ & $\begin{array}{l}\text { AGR } \\
(\%)\end{array}$ & Total & $\begin{array}{c}\text { Porcentaje de } \\
\text { Zonas censales } \\
\text { del AMS }\end{array}$ \\
\hline Clúster 1 & 8,5 & 7,4 & 2,1 & 0,7 & 0,8 & 0,3 & 0,2 & 4,8 & 3,3 & 2,2 & 8,5 \\
Clúster 2 & 21,1 & 33,8 & 13,8 & 6,3 & 5,1 & 1,1 & 1,3 & 6,8 & 3,4 & 8,0 & 21,1 \\
Clúster 3 & 8,8 & 17,2 & 11,9 & 8,7 & 6,1 & 2,5 & 3,3 & 2,7 & 2,5 & 7,9 & 8,8 \\
Clúster 4 & 19,1 & 22,3 & 29,5 & 29,8 & 23,7 & 16,5 & 22,1 & 13,3 & 13,5 & 23,0 & 19,1 \\
Clúster 5 & 21,4 & 13,3 & 26,8 & 32,9 & 33,3 & 34,5 & 37,8 & 28,7 & 26,2 & 32,0 & 21,4 \\
Clúster 6 & 21,0 & 6,0 & 16,0 & 21,5 & 31,0 & 45,1 & 35,3 & 43,6 & 51,3 & 26,9 & 21,0 \\
Total & 100 & 100 & 100 & 100 & 100 & 100 & 100 & 100 & 100 & 100 & 100 \\
\hline
\end{tabular}

Fuente: Elaboración propia en base a datos censales de 2002.

basados en la categoría ocupacional, permite generar un mapa del AMS que se observa en la Figura $\mathrm{N}^{\circ} 4$.

Cada clúster representa una complejidad interna que permite un análisis más allá de las características prevalentes. Por ejemplo, el clúster 1 es homogéneo y concentrado en cuanto a ciertas categorías dirigenciales y profesionales, pero está compuesto también por un importante porcentaje de trabajadores no calificados, lo que ayuda a comprender 
Figura $\mathrm{N}^{\circ} 4$

Mapa de tipologías socioespaciales en el AMS, por zona censal (2002)

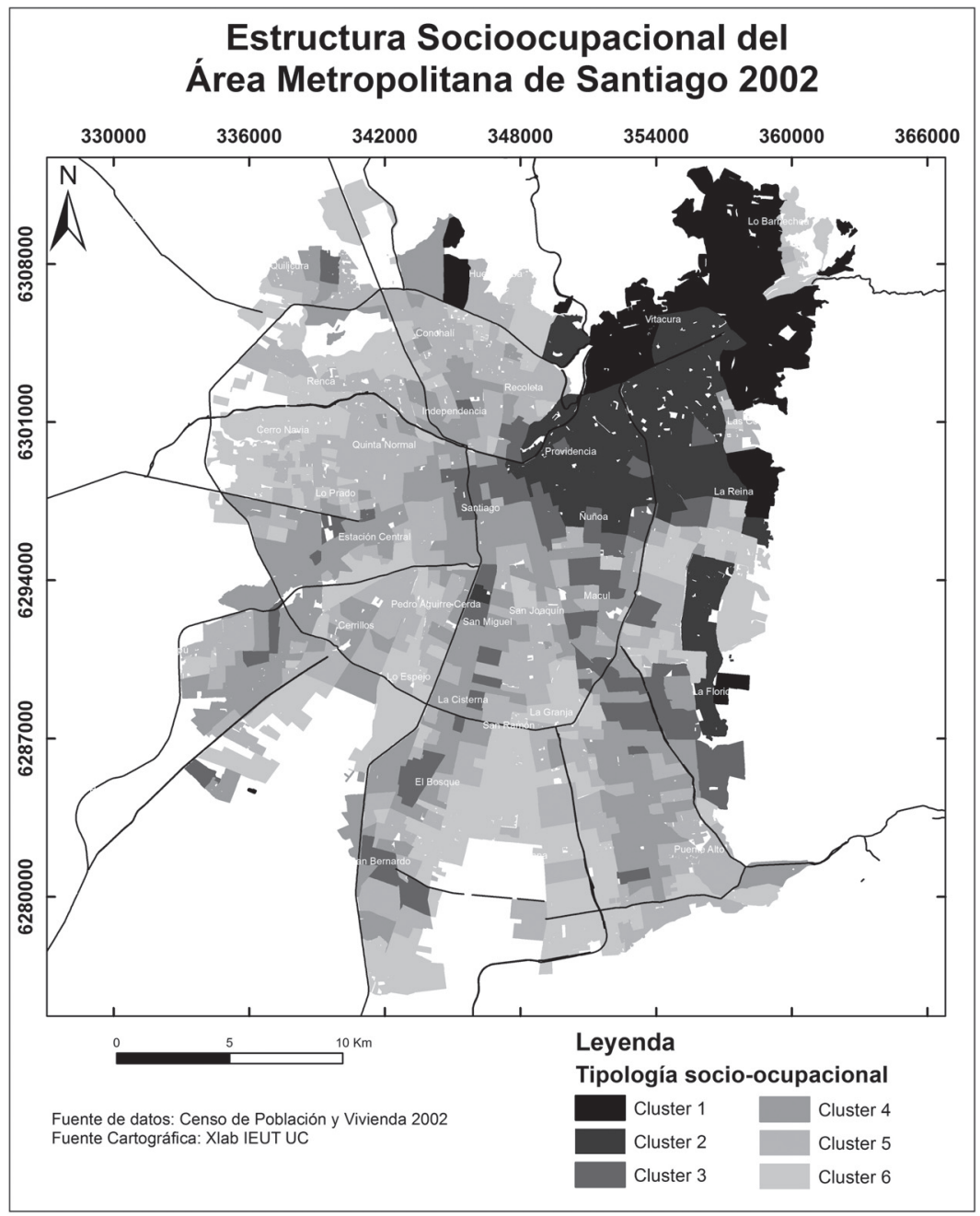

Fuente: elaboración propia en base a datos censales de 2002.

la geografía social del AMS en zonas de las comunas como Vitacura y Lo Barnechea, que son diferentes a comunas de alta renta como Providencia y Las Condes. Es decir, no se trata solo de las categorías predominantes en el territorio, sino más bien de la estructura y composición social del área metropolitana.

Como se puede observar, el resultado muestra claramente la coherencia de las características de cada grupo, en relación con su ubicación histórica en el AMS, específicamente, los grupos más altos de la jerarquía ocupacional desplegados en el llamado cono de alta renta de Santiago. Sin embargo, la clasificación generada por la construcción de tipologías, muestra también el surgimiento de algunas zonas no tradicionales. Principalmente dos fenómenos: lo que Sabatini et al. (2008) Ilaman la dispersión espacial de la elite y lo que De Mattos et al. (2005) constataron como "la progresiva expansión de los grupos medios en el área central (...) la fuerte disminución de los distritos "obreros" en prácticamente toda la mancha urbana (...) y la expansión generalizada en la ciudad 
de la presencia relativa de los trabajadores del comercio y servicios". La diferencia de este resultado con los de las investigaciones anteriores, tiene que ver con la posibilidad de caracterizar cada grupo, más allá de la presencia relativa de cada categoría en determinadas zonas. Es decir, que al observar cada tipología podemos ver que las zonas de alta renta correspondientes al grupo 1, también están constituidas por casi un 30\% de trabajadores no calificados y un $11 \%$ de técnicos, entre otros porcentajes menores de todas las categorías. Finalmente, esto nos permite realizar un análisis más detallado de la distribución socioespacial basada en las ocupaciones, para lograr determinar posibles procesos de fragmentación social en esta distribución.

\section{Conclusiones}

Finalmente, los resultados del análisis factorial de correspondencias en función de la categoría ocupacional como variable y la posterior construcción de tipologías socioespaciales se basa, al igual que los resultados de los análisis de segregación residencial, en la localización residencial de los grupos sociales, permitiendo reflejar ciertos patrones bastante marcados de distribución en el AMS. En general, todas las aproximaciones e independientemente de la escala de análisis, destacan la mayor presencia de grupos altos en el tradicional cono de alta renta de Santiago y la existencia de importantes áreas de concentración de los grupos más bajos en la periferia, principalmente en algunas comunas del sur de la ciudad. Sin embargo, es importante tener en cuenta algunas diferencias metodológicas relevantes, consideradas como un aporte de la metodología propuesta: En primer lugar, el análisis de las tipologías socioespaciales permite expresar de mejor forma la composición social de cada subunidad territorial (en este caso zonas censales), pues hace referencia permanentemente a la complejidad de grupos sociales existentes al interior de cada unidad. Por el contrario, los resultados provenientes del análisis de segregación residencial generalmente se refieren a una distribución de frecuencias simples que derivan en la representación espacial del fenómeno, a través de mapas sobre el porcentaje de prevalencia de determinado grupo social en cada subunidad territorial.
En otras palabras, mientras en el caso de las tipologías un determinado color en el mapa significa que esa subunidad territorial se asemeja a una composición social tipo en la que sabemos qué porcentaje representa cada grupo social, en los estudios tradicionales sobre segregación un determinado color en el mapa solo significa que la prevalencia de un grupo social particular en esa subunidad territorial se encuentra dentro de un rango determinado y arbitrario, desconociéndose cuáles son los otros grupos sociales presentes en ella y en qué porcentaje están representados.

Por lo tanto, el análisis de tipologías socioespaciales constituye una herramienta útil para dar cuenta del tipo de diversidad social que caracteriza a cada subunidad territorial de la ciudad, permitiendo por ejemplo, identificar aquellos espacios de mayor homogeneidad social.

En segundo lugar, ambas aproximaciones utilizan diferentes variables. Mientras las tipologías socioespaciales se han aplicado a partir de una estratificación social proveniente de la variable ocupacional, el estudio de la segregación residencial en Latinoamérica ha intentado una medición a partir del nivel socioeconómico de los hogares (Rodríguez, 2001), para lo que se han utilizado diferentes variables, principalmente el nivel educacional del jefe de hogar y los grupos socioeconómicos provenientes de los estudios de mercado. Sin embargo, estas categorías se encuentran sujetas a importantes grados de variabilidad en el tiempo, mientras el trabajo, medido a través de la categoría ocupacional es más estable.

En este contexto, la esfera del trabajo, a pesar de su construcción heterónoma, contribuye a observar las tendencias actuales en el funcionamiento y estructuración de la sociedad (Offe, 1992). Es decir, que a pesar de que el mundo del trabajo presenta un proceso de transformación profundo, asociado a los cambios en la economía y la organización de la producción, así como a los cambios socioculturales en un período de modernidad avanzada (Stillerman, Link, Cárdenas, 2012) continúa siendo, dentro de las variables disponibles al análisis, un elemento central como configurador de biografías personales y como canalizador de ciertas pautas de 
conducta, asociadas por ejemplo al uso del espacio y la conformación de una condición urbana particular.

Por último, el análisis factorial, previo a la construcción de tipologías, permite evaluar la estructura socioterritorial latente en el AMS, a partir de la distribución en el plano factorial de las diferentes categorías socioocupacionales. Esto es importante, ya que más allá de la segregación o de la composición interna de cada grupo -concentración, homogeneidad- existe una estructura latente en la distribución social del territorio. Esta estructura es evidentemente jerárquica y polarizada en Santiago. Si entendemos esta estructura como una tendencia, dada la menor "linealidad" de la distribución en períodos anteriores (Link, 2010), podemos decir que la ciudad avanza hacia una especialización social que hace difícil el encuentro con el otro y más aún, la integración social en el espacio urbano. Así, la complejidad en la investigación entre espacio físico y espacio social, a través del análisis de la diferenciación residencial, demanda un proceso de desnaturalización de la estructura urbana, vinculada a la estructura social en una sociedad capitalista.

\section{Referencias bibliográficas}

ANSELIN, L. Local indicators of spatial association-LISA. Geographical analysis, 1995, Vol. 27, No 2, p. 93-115.

ARRIAGADA, C. y MORALES, N. Ciudad y seguridad ciudadana en Chile: revisión del rol de la segregación sobre la exposición al delito en grandes urbes. EURE, 2006, Vol. 32, No 97, p. 37-48.

BAILEY, T.C. y GATRELL, A.C. Interactive spatial data analysis. Essex: Longman Scientific \& Technical, 1995.

BAROZET, E. La variable ocupación en los estudios de estratificación social. Santiago de Chile: Proyecto desigualdades, Facultad de Ciencias Sociales, Universidad de Chile. Disponible en Internet:

http://www. desigualdades. cl/wp-content/ uploads/2009/05/ocupacion.pdf
BOURDIEU, P. La miseria del mundo. Buenos Aires: Fondo de Cultura Económica, 1999.

BRENNER, N. Tesis sobre la urbanización planetaria. Nueva Sociedad, 2013, Vol. 243, p. 38-66.

BUZAI, G.D. Mapas sociales urbanos. Buenos Aires: Lugar, 2003.

CÁRDENAS, A.; LINK, F. y STILLERMAN, J. ¿Qué significa el trabajo hoy? Cambios y continuidades en una sociedad global. Santiago de Chile: Catalonia, 2012.

CASTELLS, M. La cuestión urbana. México: Siglo XXI, 2004.

DE MATTOS, C. Modernización capitalista y transformación metropolitana en América Latina: cinco tendencias constitutivas. En: GERAIGES DE LEMOS, A.I.; ARROYO, M. \& SILVEIRA, M.L. (editores). America Latina: Cidade, Campo e Turismo. São Paulo: Consejo Latinoamericano de Ciencias Sociales, 2006, p. 41-73.

DE MATTOS, C.; RIFFO, L.; YÁÑEZ, G. y SALAS, X. Reestructuración del mercado metropolitano de trabajo y cambios socioterritoriales en el Gran Santiago. Santiago de Chile: Informe de investigación proyecto FONDECYT, 2005, Vol. 1040838.

DE MATTOS, C.; FUENTES, L. y LINK, F. Tendencias recientes del crecimiento metropolitano en Santiago de Chile. ¿Hacia una nueva geografía urbana? Revista INVI, 2014, Vol. 29, No 81 , p. $193-219$.

ECHAZARRA, A. Segregación residencial de los extranjeros en el área metropolitana de Madrid. Un análisis cuantitativo. Revista Internacional de Sociología, 2010, Vol. 68, № 1, p. 165-197.

FUENTES, L. Ciudades y sociedades urbanas en transformación. Competitividad, reestructuración y cohesión social en Bogotá, Lima y Santiago en las últimas décadas. Santiago de Chile: tesis para optar al grado de Doctor en Arquitectura y Estudios Urbanos, Pontificia Universidad Católica de Chile, 2012. 
GALSTER, G.C. \& KILLEN, S.P. The geography of metropolitan opportunity: a reconnaissance and conceptual framework. Housing Policy Debate, 1995, Vol. 6, № 1, p. $7-43$

GARROCHO, C. y CAMPOS-ALANÍS, J. Réquiem por los indicadores no espaciales de segregación residencial. Papeles de Población, 2013, Vol. 19, № 77, p. 269-300.

GROISMAN, F. y SUÁREZ, A.L. Segregación residencial en la Ciudad de Buenos Aires. Población de Buenos Aires, 2006, Vol. $3, \mathrm{~N}^{\circ} 4$, p. 27-37.

HARVEY, D. The urban experience. Baltimore: Johns Hopkins University Press, 1989.

HIDALGO, R. De los pequeños condominios a la ciudad vallada: las urbanizaciones cerradas y la nueva geografía social en Santiago de Chile (1990-2000). EURE, 2004, Vol. $30, N^{\circ} 91$, p. 29-52.

KAZTMAN, R. y RETAMOSO, A. Segregación residencial en Montevideo: Desafíos para la equidad educativa. En: ILPES (editor). Documentos de trabajo del ILPES Monitor social del Uruguay. Montevideo: ILPES. Universidad Católica de Uruguay, 2006.

LEFEBVRE, H. La producción del espacio. Papers: Rrevista de Sociología, 1974, N 3, p. 219-229.

LEÓN, A. y MARTÍNEZ, J. La estratificación social chilena hacia fines del siglo XX. En: FRANCO, R.; LEÓN, A. y ATRIA, R. (editores). Estratificación y movilidad social en América Latina: transformaciones estructurales de un cuarto de siglo. Santiago de Chile: LOM Ediciones, 2007, p. 303-337.

LINK, F. Globalización, vulnerabilidad y riesgo en la nueva configuración socio-territorial del trabajo en el Área Metropolitana de Santiago. Santiago de Chile: tesis para optar al grado de Doctor en Arquitectura y Estudios Urbanos, Pontificia Universidad Católica de Chile, 2010.

MAC-CLURE, O.; BAROZET, E. y MATURANA, V. Desigualdad, clase media y territorio en Chile: ¿clase media global o múltiples mesocracias según territorios? EURE, 2014, Vol. 40, N 121, p. 163-183.

MARTORI, J.C. La segregación residencial en Barcelona. Llibre Blanc de l'Habitatge a Barcelona. Barcelona: Ajuntament de Barcelona, 2007.

MARX, K. y ENGELS, F. Manifiesto del partido comunista. Moscú: Progreso, 1970 [1848].

MASSEY, D.S. American apartheid: Segregation and the making of the underclass. Cambridge: Harvard University Press, 1993.

OFFE, C. La sociedad del trabajo: problemas estructurales y perspectivas de futuro. Barcelona: Alianza editorial, 1992.

ORGANIZACIÓN INTERNACIONAL DEL TRABAJO (OIT). CIUO: Clasificación Internacional Uniforme de Ocupaciones, 1987. Disponible en Internet: http://www.ilo.org/ public/spanish/bureau/stat/isco/isco88/major. htm

ORTIZ, J. y ESCOLANO, S. Movilidad residencial del sector de renta alta del Gran Santiago (Chile): hacia el aumento de la complejidad de los patrones socioespaciales de segregación. EURE, 2013, Vol. 39, № 118, p. 77-96.

ORTIZ, J. y SCHIAPPACASSE, P. Evolución de la diferenciación areal interna del espacio social del Gran Santiago: una dinámica opuesta a la sostenibilidad social de la ciudad. Investigaciones Geográficas, 2000, Nº 34 , p. 61-76.

PRETECEILLE, E. y DE QUEIROZ RIBEIRO, L.C. Tendências da segregação social em metrópoles globais e desiguais: Paris e Rio de Janeiro nos anos 80. EURE, 1999, Vol. 25, No 76, p. 79-102.

RIBEIRO, L.C. Metropolizacao, segmentacao socio-espacial e acumulacao urbana: as forcas da questao social no Rio de Janeiro. En El desafío de las áreas metropolitanas en un mundo globalizado. Una mirada a Europa y América Latina. Barcelona: Ed. PUC-KCB-IET, 2003. 
RODRÍGUEZ, J. Segregación residencial socioeconómica: ¿qué es?, ¿cómo se mide?, ¿qué está pasando?, ¿importa? Santiago de Chile: Serie Población y desarrollo $N^{\circ} 16$, CEPAL, 2001.

RODRÍGUEZ, J. Segregación residencial, migración y movilidad espacial. El caso de Santiago de Chile. Cadernos Metrópole N ${ }^{\circ}$ 17, 2007, p. 135-168.

RODRÍGUEZ, J. y ARRIAGADA, C. Segregación residencial en áreas metropolitanas de América Latina: magnitud, características, evolución e implicaciones de política. Santiago de Chile: Serie Población y Desarrollo N ${ }^{\circ}$ 47, CEPAL, 2003.

RUIZ-TAGLE, J. y LÓPEZ, E. El estudio de la segregación residencial en Santiago de Chile: revisión crítica de algunos problemas metodológicos y conceptuales. EURE, 2014, Vol. $40, \mathrm{~N}^{\circ} 119$, p. 25-48.

SABATINI, F. y SIERRALTA, C. Medición de la Segregación Residencial: meandros teóricos y metodológicos, y especificidad latinoamericana. Santiago de Chile: Documento de Trabajo $N^{\circ} 38$, Instituto de Estudios Urbanos y Territoriales, Pontificia Universidad Católica de Chile, 2006.

SABATINI, F.; CÁCERES, G. y CERDA, J. Segregación residencial en las principales ciudades chilenas: Tendencias de las tres últimas décadas y posibles cursos de acción. EURE, 2001, Vol. 27, № 82, p. 21-42.
SABATINI, F.; WORMALD, G.; SIERRALTA, C. y PETERS, P. Segregación residencial en Santiago: Tendencias 1992-2002 y efectos vinculados con su escala geográfica. En: SABATINI, F.; SALCEDO, R.; WORMALD, G. \& CÁCERES, G. (editores). Tendencias de la segregación en las principales ciudades chilenas. Santiago de Chile: Pontificia Universidad Católica de Chile-Instituto Nacional de Estadísticas, 2008, p. 19-41.

SHEVKY, E. \& BELL, W. Social area analysis: theory, illustrative application and computational procedures. Standford: Stanford University Press, 1955.

SIERRALTA, C. Efectos de la segregación residencial socioeconómica en los jóvenes de extracción popular en Santiago de Chile (1992-2002). En: SABATINI, F.; SALCEDO, R.; WORMALD, G. y CÁCERES, G. (editores). Tendencias de la segregación en las principales ciudades chilenas. Santiago de Chile: Pontificia Universidad Católica de Chile-Instituto Nacional de Estadísticas, 2008, p. 141-163.

TORCHE, F. y WORMALD, G. Chile, entre la adscripción y el logro. Estratificación y movilidad social en América Latina. En: FRANCO, R.; LEÓN, A. y ATRIA, R. (editores). Estratificación y movilidad social en América Latina: transformaciones estructurales de un cuarto de sig/o. Santiago de Chile: LOM Ediciones, 2007. p. 339-385.

WILSON, W.J. The Truly Disadvantaged: The Inner City, the Underclass, and Public Policy. Chicago: University of Chicago, 1987. 